\title{
Histopathological and Direct Immunofluorescent Changes in Renal Biopsies of Patients with Systemic Lupus Erythematosus
}

\author{
Rohi Wani, M.D., Azra Shah, M.D., M. Saleem Najar, M.D., Parveen Shah, M.D. \\ Department of Pathology and Nephrology, Sher-i-Kashmir Institute of Medical Sciences, Soura Srinagar, Kashmir.
}

\section{A B S T R A C T}

BACKGROUND: Renal involvement in Systemic lupus erythematosus (SLE) can display diverse morphological patterns. OBJECTIVES: To study the histopathological changes along with activity and chronicity of lesions in kidney biopsies of patients with SLE.

METHODS: Thirty-five renal biopsies of SLE patients were received during two and a half years period. The biopsies were classified according to the WHO classification. Immunofluorescence pattern was also studied and correlated with histological features.

RESULTS: There were 32(91.4\%) females and 3(8.6\%) males. Age of the subjects ranged from 15 to 60 years with a mean age of 33.48 years. The most common class of lupus nephritis was class IV which was observed in $54.29 \%$ of biopsies. Immunofluorescence was performed in 30 biopsies and all these were positive for various antisera with anti-lgG positivity in $27(90 \%)$. Mesangial deposits were seen in 13(43.3\%) cases followed by granular deposits in $9(30 \%)$ cases.

CONCLUSION: Majority of SLE cases at SKIMS present during the severe course of the disease. JMS 2012;15(2): 162-65

Key words: Systemic lupus erythematosus, lupus nephritis, renal biopsies.

Systemic lupus erythematosus (SLE) is one of the most common autoimmune disorders in women during their child bearing age and is being recognized increasingly throughout the world. The female to male ratio is 9:1 when the female is in child bearing age. The fundamental defect in SLE is failure of regulatory mechanisms to sustain self tolerance. SLE can involve any organ and the most common visceral organ involved is the kidney. Renal disease occurs approximately in half the patients with SLE as immune

\section{Correspondence:}

Dr. Rohi Wani

Senior Resident

Deptt of Pathology, Sher-i-Kashmir Institute of Medical Sciences, Srinagar, Kashmir.

E-mail:rohiwani@gmail.com mediated nephritis is a common complication. A kidney biopsy is thus essential in renal assessment of patients with SLE. Almost all patients of SLE have abnormalities of renal biopsy especially if histopathology is supplemented by immunofluorescence and electron microscopy. Renal biopsy using WHO classification provides valuable information concerning the risk of renal failure even 10 years after initial biopsy and plays a major role in management of lupus. ${ }^{1,2}$

\section{Methods}

This study was conducted on 35 percutaneous renal biopsies of SLE patients received in our department over a period of two and a half years (from Jan 2003 to June 2005). The patients coming on follow up in the department of Nephrology were also included in this study. Clinical 
features and lab parameters were recorded. The kidney biopsies were received in formalin and after processing stained with Hematoxylin and Eosin stain, Periodic acid Schiff's stain and Jones methanamine silver stain. The slides were studied in detail under the light microscope and changes were classified as per modified WHO classification of Lupus Nephritis. ${ }^{3}$ Activity and chronicity indices were also studied. ${ }^{4.5}$ Direct immunofluorescence ${ }^{6}$ was done in 30 renal biopsies as the tissue was inadequate in the remaining 5 . Sections of less than 4 microns of fresh unfixed material were stained with differential FITC (Fluorescein isothiocyanate) for IgG, IgM, IgA, C3,C4 and fibrinogen and studied under fluorescent microscope. Immunofluorescence(IF) was described according to the pattern primarily in glomerulus as mesangial, granular, lumpy and membranous according to Koffler et al. ${ }^{7}$ Mesangial deposits were characterized by irregular strands of protein lined between capillary loops within the mesangial cells or in the mesangial matrix. Linear deposits appeared as fine, homogeneous, continuous fluorescence along the glomerular basement membrane. Granular deposits had a punctuate pattern of fluorescence comprising of granules which varied in size and were distributed irregularly throughout the glomerulus. Lumpy deposits were coarse and obscured the normal architecture of glomeruli.

\section{Results}

The age range in our study varied from 15 to 60 years with maximum number of patients i.e., $28(80 \%)$ in the age group of 21- 40 years. Only one patient with lupus nephritis

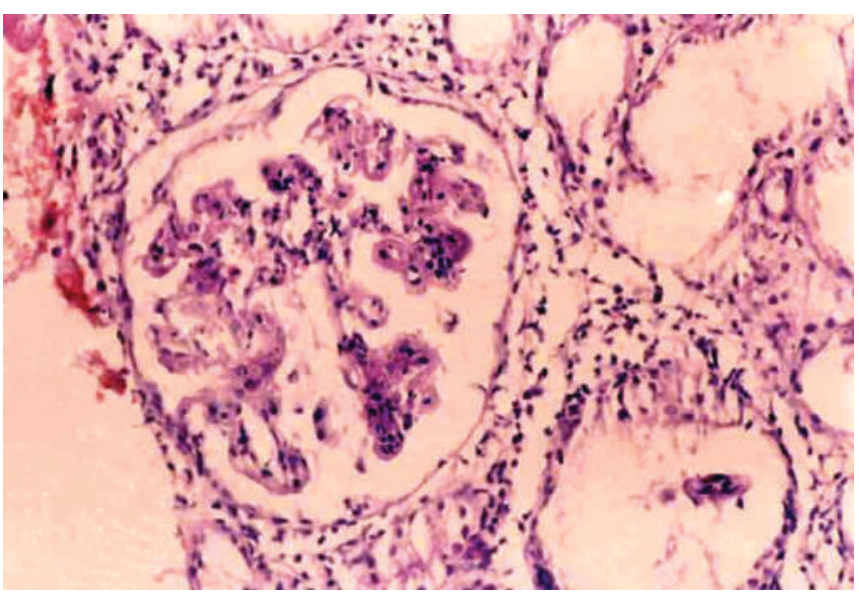

FIGURE 1. Photomicrograph of lupus nephritis-class IV showing mesangial prominence with wire loop lesions of capillary BM. $(\mathrm{H} \& \mathrm{E}, \mathrm{X} 400)$.

presented at the age of 15 years. Out of 35 patients 32 were females and 3 males. The histological findings of lupus nephritis fell into five classes from class I to V (Figure 1-2). There was no case in class VI. Of these 35 patients 2,3,5,19 and 6 were in class I to $\mathrm{V}$ respectively (Table 1). Presence or absence of activity and chronicity was studied in all the

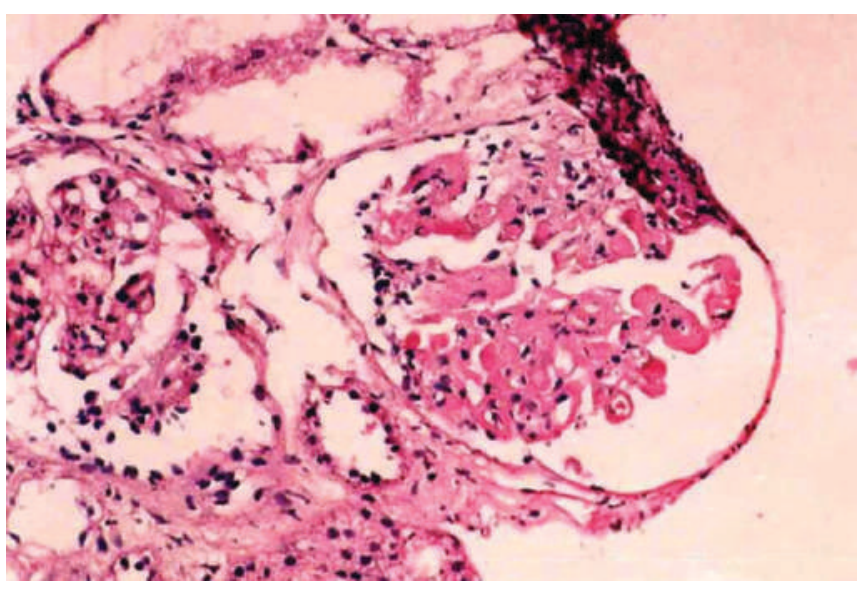

FIGURE 2. Photomicrograph of membranoproliferative glomerulonephritis showing mesangial prominence with wire loop lesions and intraluminal hyaline thrombi. (PAS.x400).

TABLE 1. Class distribution of lupus nephritis according to WHO classification

\begin{tabular}{lcc} 
Class & No. of cases & \%age \\
I & 2 & 5.71 \\
II & 3 & 8.57 \\
III & 5 & 14.29 \\
IV & 19 & 54.29 \\
V & 6 & 17.14 \\
VI & - & 0 \\
\hline Total & 35 & 100 \\
\hline
\end{tabular}

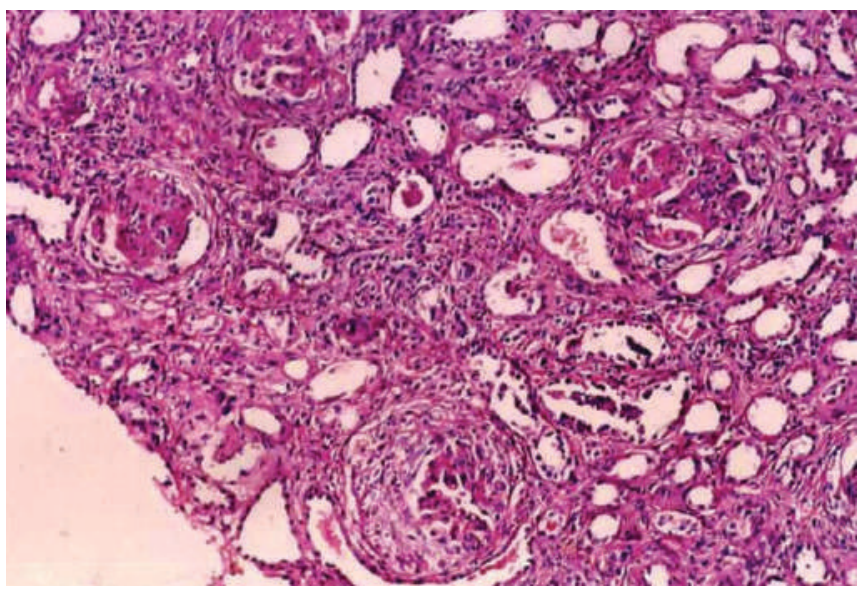

FIGURE 3. Photomicrograph showing crescent formation, fibrinoid necrosis of glomerular tuft, tubular atrophy and interstitial inflammation.(H\&E,x10sO).

biopsies (Figure 3-4). There were no active or chronic lesions in class I. In class II active lesions were seen in $33 \%$ of cases, whereas they were seen in $100 \%$ of class III and $94.7 \%$ of class IV biopsies. Chronic lesions were most common in class III and IV as $40 \%$ and $73 \%$ respectively (Table 2 ).

Immunofluorescence was positive in all the 30 biopsies including 2 cases of class I which were normal on histopathology. Anti IgG positivity was seen in 27/30 (90\%) of biopsies followed by anti IgM in 21/30 (70\%), IgA in13/30 


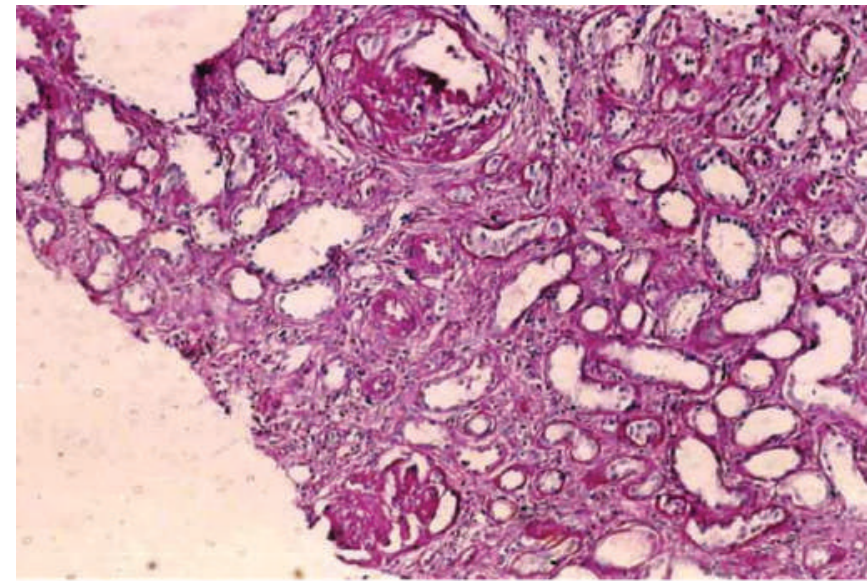

FIGURE 4. Photomicrograph showing glomerular sclerosis, fibrous crescent formation, tubular atrophy and leucocytic infiltration.(PAS,x100).

TABLE 2. Activity and chronicity of lesions in relation to WHO class in renal biopsies

\begin{tabular}{lccc} 
WHO Class & No. of biopsies & Active lesions $(\%)$ & Chronic lesions $(\%)$ \\
\hline I & 2 & $0(0)$ & $0(0)$ \\
II & 3 & $1(33)$ & $0(0)$ \\
III & 5 & $5(100)$ & $2(40)$ \\
IV & 19 & $18(94.7)$ & $4(73.6)$ \\
V & 6 & $2(33.3)$ & $3(50)$ \\
\hline
\end{tabular}

(43.33\%), C3 in 2/30 (6.67\%), C4 in17/30 (56.6\%) and fibrinogen in $8 / 30(26.66 \%)$. Often the study revealed more than one class of immunoglobulin in a coarse, granular pattern in the mesangium and peripheral capillary walls in all glomeruli. Four distinct patterns of glomerular staining were observed namely, mesangial, granular, lumpy and membranous. Mesangial deposits were the most common pattern seen in predominantly 13 cases out of a total of 30 . Predominantly granular pattern was seen in 9 cases with lumpy deposits in 5 and membranous deposits in 3 cases. Out of the 13 predominantly mesangial deposits 2 were from class I, 3 from class II, 1 from class III and 7 from class IV. Out of 9 granular patterns 6 were from class IV lupus nephritis. Lumpy deposits were shown by one case of class III and four cases of class IV lupus nephritis. Purely membranous pattern was seen in three cases out of five cases of class V lupus nephritis.

\section{Discussion}

Lupus nephritis (LN) is a frequent and potentially serious complication of SLE. Renal involvement often develops concurrently and shortly following the onset of SLE and usually correlates well with the onset of glomerular involvement.

In our study $80 \%$ of patients were in the age group of 21 to 40 years that is active reproductive age group with mean age of presentation of 33.48 years. Females outnumbered males by a ratio of 10.67:1. The age range varied from 15 to 60 years. This co-relates well with most other studies from India, however mean age of presentation was more in our study.

The most common class was class IV which accounted for $54.29 \%$ of cases followed by class $V$ which accounted for $17.14 \%$ cases. In class I ( 2 cases), there was no light microscopic abnormality in renal biopsies but both the cases showed mesangial deposits of IgG and C3. Mesangial (class II) LN was diagnosed in 3 patients where increase in mesangial matrix and cellularity was seen. Focal proliferative glomerulonephiritis was present in 5 patients. Changes of diffuse proliferative glomerulonephiritis showed widespread involvement of glomerulus with capillary endothelial and mesangial cell proliferation along with interstitial and tubular inflammation in most of the cases. Other workers like Pollack, Baldwin and Cameron ${ }^{8}$ also found class IV as the commonest class of lupus nephiritis. Shete et al ${ }^{9}$ studied 27 renal biopsies of LN in which class IV was observed in maximum number of cases i.e, 10 cases. Similarly Jindal et $\mathrm{al}^{10}$ while studying fatal complication of SLE in 25 cases observed that renal involvement was invariably present in $96 \%$ of cases with diffuse proliferative glomerulonephiritis being the commonest lesion (60\%). Rapidly progressive glomerulonephritis was seen in a total of 7 cases. All these cases were from Class IV.

Activity and chronicity of the lesions was done according to Austin et al. ${ }^{4,5}$ There were no active or chronic lesions in class I. In class II active lesions were seen in $33 \%$ of cases due to interstitial inflammation. In Class III and IV, almost all of the cases showed active lesions primarily in the glomerulus as end capillary proliferation. There was no chronic lesion in Class I and II. Chronic lesions were most common in Class III and IV as $40 \%$ and $73 \%$ respectively. This group of class showed a rapid and progressive loss of renal function in accordance with other studies, accompanied by hematuria, proteinuria, oliguria and rapid clinical deterioration. Our study was in accordance with that of Gladman et al ${ }^{11}$ who found both active and chronic lesions to be most common in Class III and Class IV i.e., proliferative group of glomerulonephritis in a study of 148 kidney biopsies. They also found active lesions in $17 \%$ \& $8 \%$ of class I and class II LN which was due to interstitial inflammation. In class $\mathrm{V}$ there were $18 \%$ of cases showing both active and chronic lesions whereas in our study $33 \%$ and $50 \%$ showed active and chronic lesions in class $\mathrm{V}$.

Immunofluorescence was positive in all the 30 biopsies, including 2 cases of Class I which were normal on histopathology. Mesangial deposits were the most common predominant pattern seen in 13 out of 30 cases. Infact, mesangial positivity was seen in almost all the cases with or without other patterns (primarily in the glomerulus). Predominantly granular pattern was seen in 9 cases with 
lumpy deposits in 5 and membranous in 3 cases. On over all evaluation granular and lumpy deposits were seen in proliferative class of lupus nephritis whereas membranous pattern was predominantly seen in class Vlupus nephritis.

This evaluation is consistent with Hill and Hinglas ${ }^{12}$, and Koffler et $\mathrm{al}^{7}$ wherein they found that solitary mesangial deposits without histological evidence of glomerular injury whereas granular deposits were associated with the appearance of glomerulonephritis. These studies have also substantiated that the initial deposits of immunoglobulins and complement is located on the mesangium and the presence of proteinuria is associated with granular deposits in early stage of disease with lumpy deposition in more advanced cases of proliferative glomerulonephritis. Thus whereas presence of mesangial deposits are frequent, the appearance of granular deposits should be considered evidence of significant renal injury. Banff et $a l^{13}$ in a study of 147 patients with lupus nephritis found similar features. They found mesangial pattern mainly in class II with a few examples in class I and class III, the peripheral pattern (lumpy and granular) was by far the most common in class IV and most common in class VI whereas membranous pattern was a rule in class $\mathrm{V}$.

In context to the type of immunodeposition with antisera anti IgG positivity was most common (90\%), followed by anti $\operatorname{IgM}(70 \%)$ and anti C3 positivity (56.6\%). Full house effect-with the presence of $\operatorname{IgG}, \operatorname{IgM}$ and $\operatorname{IgA}$ positivity in the same biopsy was seen in 5 out of 30 biopsies. These findings were similar to other studies by Cameron, Banff and Gadgil. ${ }^{8,913}$ In our study both the complement components and immunoglobulins were present in the same biopsy at the same time. Full house effect with the presence of IgG, IgM and IgA was seen in 5 out of 30 biopsies. C4 was seen as the least common immonofluorescence, in only 2 cases. Although Banff et al ${ }^{13}$ found IgA particularly rare in class I, II and $\mathrm{V}$ but no such correlation was found in our study.

Anemia was a fairly constant finding along with proteinuria which was seen in almost all the cases. The severity of proteinuria correlated well with the severity of histological findings. Among the routine lab investigations serum creatinine correlated well with the severity of the disease. All the cases were positive for antinuclear antibody at any point of time with ds DNA positivity in $57.14 \%$ of cases. After fever, dermatologic symptoms were the most common mode of presentation among other associated features.

In conclusion, the most common class of Lupus nephritis in our study was diffuse proliferative glomerulonephritis (54.29\%) with mean age at presentation of 33.48 years implying that most cases present during the severe course of the disease. Most of the cases were positive for anti
IgG on immunofluorescence with mesangial pattern being the most common pattern. Lumpy and granular deposits were primarily seen in proliferative class of lupus nephritis.

\section{References}

1. Kumar V, Abbas AK, Fausto N. Robbins and Cotran. Pathologic basis of disease. 7th ed. WB Saunders Company, 2004:227-35.

2. Brenner BM. Brenner and Rector's. The Kidney. Vol-I. 7thed. Saunders, 2004:1382-93.

3. Seshan SV. Renal lesions in systemic lupus erythematosus: An update. IX International CME on Surgical Pathology and Cytology; 2004: 27-41.

4. Calvin RB, Bhan AK, Robert T. Diagnostic immunopathology. 2nd ed. Raven Press, 1994:623-45.

5. Austin HA, Muenz LR, Joyce KM, et al. Prognostic factors in lupus nephiritis: contribution of renal histological data. Am J Med 1983;75:382-391.

6. Austin HA, Muenz LR, Joyce KM, Antonovych TT, Balow JE. Diffuse proliferate lupus nephritis. Identification of specific pathologic features affecting renal outcome. Kidney Int 1984;25: 689-95.

7. Koffler D, Agnello V, Carr RI, Kunkel HG. Variable patterns of immunoglobulin and complement deposition in the kidney of patients with systemic lupus erythematosus. Am JMed 1969; 56:305-12.

8. Cameron JS, Turner DR, Ogg CS, et al. Systemic lupus with nephritis: a long term study. QJMed 1979;48:1-24.

9. Gadgil NM, Ranadev NV, Shete M, Bhatia S. Clinical, morphological, immunological correlation of kidney biopsies and prognostication. Indian J Pathol Microbiol 1999; 42:3-10.

10. Jindal B, Joshi K, Radotra BD, Banerjee AK. Fatal complications of systemic lupus erythematosus- an autopsy study from north India. Indian J Pathol Microbiol 2000; 43:311-317.

11. Gladman DD, Urowitz MB, Cole E, Ritche S, Chang C, Churg J. Kidney biopsy in SLE I: a clinicalmorphologic evaluation. QJMed 1989; 272:1125-33.

12. Hill GS, Hinglais N, Tron F, Bach JF. Systemic lupus erythematosus: morphologic correlations with immunologic and clinical data at the time of biopsy. Am JMed 1978; 64: 61-79.

13. Banfi G, Mazzucco G, Belgiojoso GB et al. Morphological parameters in lupus nephritis: their relevance for classification and relationship with clinical and histological findings and outcome. QJ Med 1985;217: 153-68. 